\title{
Challenging the Treatment Paradigm: Selecting Patients for Surgical Management of Hepatocellular Carcinoma with Portal Vein Tumor Thrombus
}

\section{Nicole M Nevarez (D) Adam C Yopp}

Department of Surgery, Division of Surgical Oncology, University of Texas Southwestern Medical Center, Dallas, TX, 75390, USA
Correspondence: Adam C Yopp Department of Surgery, Division of Surgical Oncology, University of Texas Southwestern Medical Center, 5323

Harry Hines Boulevard, Dallas, TX, 75390 , USA

Tel $+1214648-5870$

$\mathrm{Fax}+1214648-1118$

Email adam.yopp@utsouthwestern.edu

\begin{abstract}
Portal vein tumor thrombus (PVTT) remains a common presentation in patients with hepatocellular carcinoma (HCC). Approximately $30-50 \%$ of patients newly diagnosed with HCC will present with a concomitant PVTT. Current guidelines recommend systemic therapy for treatment of HCC with PVTT. Real-world application of partial hepatectomy in HCC patients with PVTT has increased over the past two decades, as perioperative complications have declined. However, it is unclear if there is an association between the extent of PVTT and overall survival and rates of recurrence and whether the perioperative morbidity outweighs these potential benefits. Partial hepatectomy with en bloc resection of PVTT in second-order branches and distal can offer significant benefits in carefully selected patients; however, once the HCC-associated PVTT extends into first-order portal venous branches or more proximal into the superior mesenteric vein, the risks of surgical resection outweigh the benefits. The aim of this review is to determine which patients with HCC presenting with PVTT benefit from surgical resection. We will discuss the classification systems of PVTT and review both outcome and perioperative measures in patients undergoing partial hepatectomy with extirpation of HCC-related PVT.
\end{abstract}

Keywords: hepatocellular carcinoma, portal vein tumor thrombus, liver cancer

\section{Introduction}

Hepatocellular carcinoma (HCC) is the third leading cause of cancer-related deaths worldwide and the fastest-rising cause of cancer mortality in the United States. ${ }^{1,2}$ Commonly occurring in a background of chronic liver disease with or without underlying cirrhosis HCC prognosis is dependent not only on tumor burden, but on underlying liver function and patient performance status. Surgical resection consisting of partial hepatectomy, liver transplantation, or ablative therapies remain the only curative options in HCC treatment with five-year survival rates in selected patients approaching $60 \%$ worldwide. ${ }^{3}$ Due to heterogeneity of the patient population and low utilization of HCC screening, only $10-37 \%$ of patients are candidates for surgical resection at initial diagnosis. ${ }^{4-6}$

HCC has a known predilection for macrovascular invasion with tumor invasion and ingrowth into the hepatic and/or portal venous systems. Portal vein tumor thrombus (PVTT) is a relatively common presentation, observed in $30-50 \%$ of 
patients with newly diagnosed $\mathrm{HCC}^{7}$ PVTT portends a poor prognosis due to 1) associated intra- and extrahepatic tumor dissemination via hematogenous tumor spread and 2) the sequelae of elevated portal venous pressures with associated clinical manifestations of portal hypertension.

According to the Barcelona Clinic Liver Cancer (BCLC) classification endorsed by both the American and European Associations for the Study of Liver Disease (AASLD and EASL) as both a staging and treatment algorithm, treatment for PVTT (BCLC stage C) historically has included systemic therapy regimens including tyrosine kinase inhibitors or more recently combination immunotherapeutics. ${ }^{8-11}$ Even with the development of novel systemic regimens, the median overall survival time in patients diagnosed with PVTT is generally less than one year. ${ }^{12}$ However, this paradigm has recently been challenged with the real-world application of surgical resection in HCC patients with PVTT by experienced centers from both Western and Eastern countries with low perioperative complications and five-year overall survival rates greater than $35 \%$ in carefully selected patients. $^{13}$

The aim of this review is to provide evidence to support surgical resection consisting of partial hepatectomy in patients with PVTT. We will discuss PVTT classification systems, extent of surgical resection and associated prognosis by degree of PVTT and criteria used to select patients with HCC-related PVTT for surgical resection.

\section{Classification Systems of Portal Venous Tumor Thrombus}

To ensure a balanced comparison between studies describing surgical resection for patients with HCC-related PVTT, it is paramount to understand the varying classification systems describing the extent of PVTT. Although there is no universally accepted PVTT classification system we herein describe the three most commonly utilized: 1) Liver Cancer Study Group of Japan classification (LCSGJ), 2) Cheng classification and 3) $\mathrm{Xu}$ classification. ${ }^{14-16}$

\section{Liver Cancer Study Group of Japan Classification}

The Liver Cancer Study Group of Japan is one of the earliest described PVTT classification systems but since its initial description has been modified accordingly. ${ }^{14}$ In the latest iteration of their General Rules for the Clinical and Pathological Study of Primary Liver Cancer they divide PVTT in the presence of macroscopic HCC into five overall types (Figure 1):

- Vp0: Absence of PVTT

- Vp1: PVTT distal to, but not in, the second-order branches of the left and right portal vein

- Vp2: PVTT in the second-order branches of the portal vein

- Vp3: PVTT in the first-order branch of the portal vein

- Vp4: PVTT in the main trunk of the portal vein or PVTT in contralateral portal vein branch, or both

\section{Cheng Classification}

In 2007, Shi et al first described a PVTT staging system based not only on macroscopic PVTT but also incorporated microscopic PVTT (Figure 2). ${ }^{15}$

- Type 0: PVTT seen only on microscopy.

- Type I: PVTT in the segmental branches of the portal vein or above.

- Type Ia: PVTT involving segmental branches of the portal vein or above,

○ Type Ib: PVTT involving segmental branches of the portal vein extending to sectoral branches.

- Type II: PVTT in the right or left portal vein.

○ Type IIa: PVTT involving the right/left portal vein,

○ Type IIb: PVTT involving both the right and left portal vein.

- Type III: PVTT in the main portal vein trunk.

- Type IIIa: PVTT involving the main portal vein trunk for no more than $2 \mathrm{~cm}$,

- Type IIIb: PVTT involving the main portal vein trunk for more than $2 \mathrm{~cm}$.

- Type IVa: PVTT in the superior mesenteric vein.

\section{Xu Classification}

$\mathrm{Xu}$ et al simplified the classification system for PVTT even further than the LCSGJ and Cheng classification systems. ${ }^{16}$ They divided patients with HCC related PVTT into two groups (Figure 3).

- Group A: Involvement of main trunk of the portal vein trunk or both the left and right portal veins 


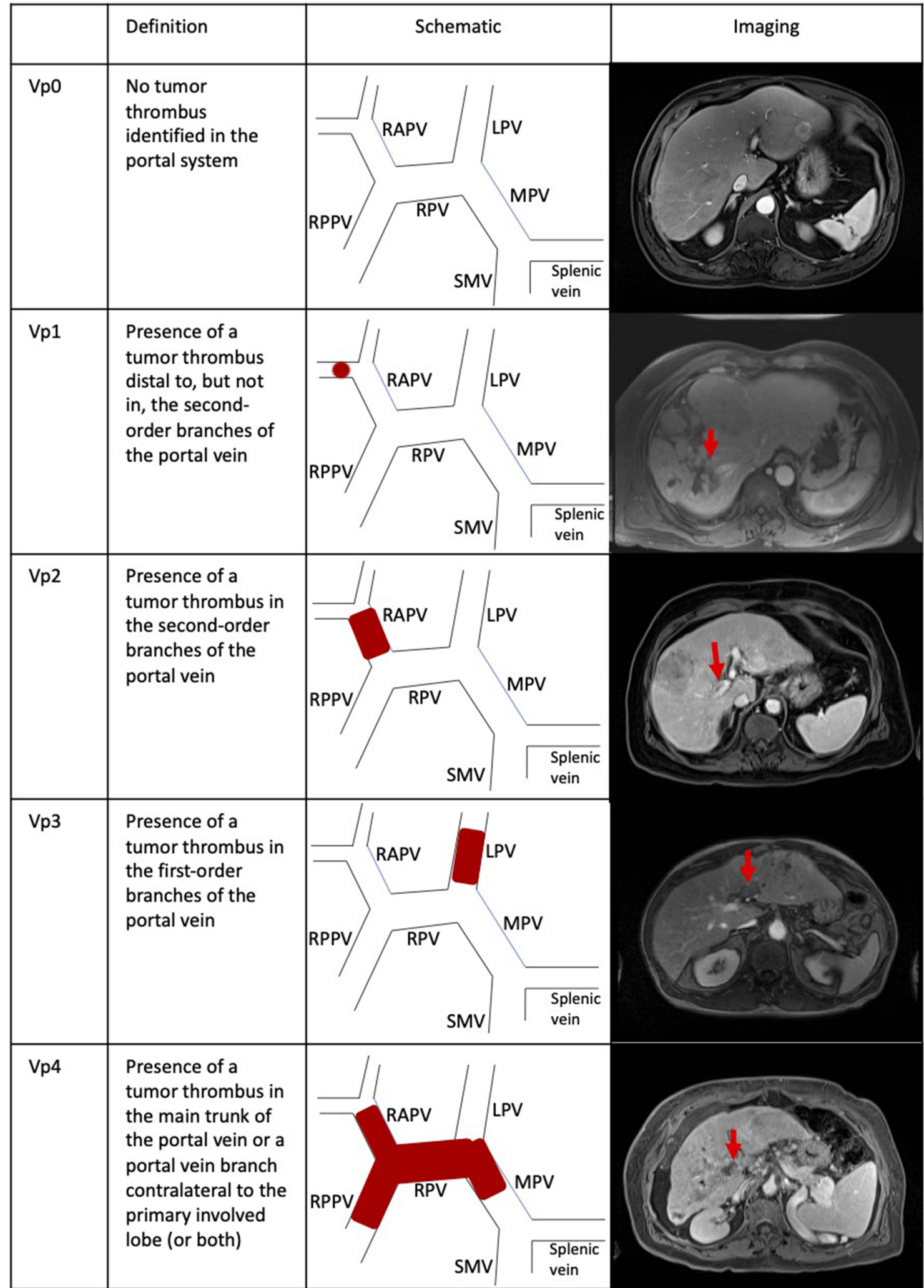

Figure I Liver Cancer Study Group of Japan PVTT classification system with the definition for each type followed by corresponding schematic and imaging. Abbreviations: RPPV, right posterior portal vein; RAPV, right anterior portal vein; RPV, right portal vein; LPV, left portal vein; MPV, main portal vein; SMV, superior mesenteric vein. 


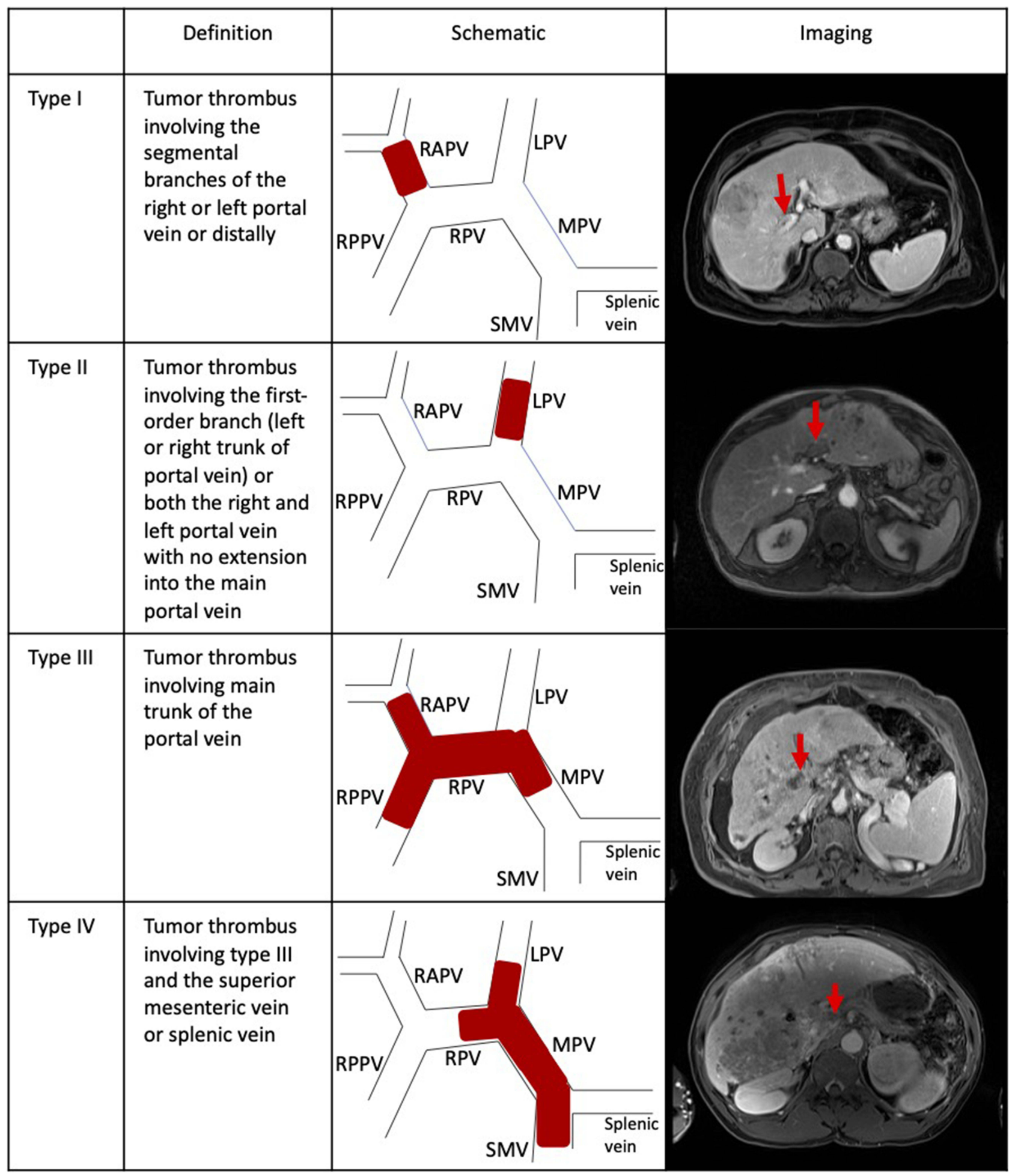

Figure 2 Cheng's classification system with the definition of each type followed by corresponding schematic and imaging.

Abbreviations: RPPV, right posterior portal vein; RAPV, right anterior portal vein; RPV, right portal vein; LPV, left portal vein; MPV, main portal vein; SMV, superior mesenteric vein. 


\begin{tabular}{|c|c|c|c|c|}
\hline & Definition & Schematic & & Imaging \\
\hline Type A & $\begin{array}{l}\text { Tumor thrombus } \\
\text { involving the main } \\
\text { portal venous } \\
\text { trunk or both the } \\
\text { right and left } \\
\text { portal veins }\end{array}$ & $\begin{array}{l}\text { V } \\
\text { RPV }\end{array}$ & $\begin{array}{l} \\
\begin{array}{l}\text { Splenic } \\
\text { vein }\end{array}\end{array}$ & \\
\hline Type B & $\begin{array}{l}\text { Tumor thrombus } \\
\text { of only the left or } \\
\text { right portal vein }\end{array}$ & $\begin{array}{l}\text { PV } \\
\text { RPV }\end{array}$ & $\begin{array}{l}\text { V } \\
\begin{array}{l}\text { Splenic } \\
\text { vein }\end{array}\end{array}$ & \\
\hline
\end{tabular}

Figure 3 Xu's classification system with the definition for each type followed by corresponding schematic and imaging.

Abbreviations: RPPV, right posterior portal vein; RAPV, right anterior portal vein; RPV, right portal vein; LPV, left portal vein; MPV, main portal vein; SMV, superior mesenteric vein.

- Group B: Involvement of only the left or right portal vein.

For the purposes of this review, Vp1 and $\mathrm{Vp} 2$ are included in type I PVTT. Vp3, type II, and group B all encompass PVTT in the first branch also named the right or left portal vein. Similarly, Vp4, type III, and group A encompass PVTT that has invaded the main portal vein or affects a branch on the opposite side. Type IV of the Cheng classification system does not have any counterparts and is the only category in the classification systems to include PVTT extending to the superior mesenteric vein (SMV) and the inferior vena cava (IVC).

There are significant limitations to the aforementioned three PVTT classification systems including that all three are strictly anatomical in nature with regard to the portal venous system and fail to take into consideration underlying liver function or extent of tumor involvement throughout the hepatic parenchyma.

\section{Extent of Surgical Resection According to Degree of Portal Venous Tumor Thrombus}

Surgical resection in the form of partial hepatectomy in patients with HCC and associated PVTT was first described nearly forty years ago. ${ }^{17}$ Although improvements in surgical technique and perioperative care has dramatically improved, outcomes following hepatic resection, patients presenting with PVTT secondary to HCC have significantly worse outcomes than in the absence of PVTT. ${ }^{12,18}$ The overall goal of partial hepatectomy in patients with $\mathrm{HCC}$ and associated PVTT includes not only margin negative tumor resection but also thrombus extirpation within the portal system to prevent the downstream sequelae of portal hypertension and potential hematogenous tumor dissemination. As such, the surgical options for liver resection in patients with concomitant PVTT include: 1) partial hepatectomy with en bloc resection of ipsilateral tumor thrombus, 2) partial hepatectomy with en bloc vascular resection and 
reconstruction or 3) partial hepatectomy with tumor thrombectomy.

\section{Portal Venous Tumor Thrombus Within Extent of Hepatic Parenchymal Resection}

For patients presenting with HCC-related PVTT with LCSGJ Vp1-2 or Cheng's classification Group 1 (PVTT limited to second-order or segmental portal branches or more distal), partial hepatectomy with en bloc resection of ipsilateral tumor thrombus is appropriate. Theoretically, patients presenting with Vp3 PVTT could undergo surgical resection in a similar fashion, however the high likelihood of a R1 or R2 resection should preclude this as a viable option.

Along the continuum of extent of PVTT in HCC, patients with portal venous involvement of second- and third-order branches have the best prognosis and receive the most benefit from liver resection compared to more extensive involvement of portal system by tumor thrombus (Table 1). Kudo et al retrospectively examined 20,850 patients at 482 institutions within Japan. ${ }^{19}$ Patients treated with liver resection with $\mathrm{Vp} 1$ and $\mathrm{Vp} 2$ had 1-, 3-, and 5-year survival rates of $83.8 \%, 62.3 \%, 50.7 \%$ and $67.3 \%, 41.2 \%$, $33.1 \%$, respectively. $\mathrm{Vp} 1$ and $\mathrm{Vp} 2$ patients had median OS at 61.2 and 25.9 months, respectively. Kokudo et al demonstrated that overall and disease-free survival rates are associated with the extent of PVTT. ${ }^{20}$ The median survival time after partial hepatectomy with en bloc PVTT resection was
4.13 years (95\% CI 3.40-5.81) and 2.49 years $(95 \%$ CI 1.92-3.08) in Vp1 and Vp2 patients, respectively. The recurrence free survival following surgery in $\mathrm{Vp} 1$ and $\mathrm{Vp} 2$ patients was 1.23 years $(95 \% \mathrm{CI} 1.04-1.73)$ and 0.82 years (95\% CI $0.65-1.05)$, respectively. In both $\mathrm{Vp} 1$ and $\mathrm{Vp} 2$ patients the most frequent site of recurrences were intrahepatic in nature. The post-operative 90-day mortality rate increased according to the extent of PVTT: Vp1 2.4\%; Vp2 3.0\%. In a similar fashion, Shi et al demonstrated that in patients with Type I PVTT, three-year overall survival was $25 \%$ with no evidence of post-operative mortality and intrahepatic location remain the most common site of recurrence following partial hepatectomy. ${ }^{21}$

Although partial hepatectomy with en bloc PVTT resection is an appropriate surgical option for HCC patients with PVTT distal to and including second-order portal branches, due to high recurrence rates secondary to poor tumor biology, careful patient selection is necessary to determine the optimal patient population for surgical resection. Zhang et al recently, in a multicenter study, developed an externally validated scoring system to examine in a subset of HCC patients with PVTT limited to second-order and distal portal branches when partial hepatectomy with en bloc PVTT resection would offer a significant outcome benefit. ${ }^{22}$ The scoring system included total bilirubin, alpha-fetoprotein, tumor diameter, and presence of satellite lesions. A score of $>3$ reliably identified (AUCs 0.68-0.72) HCC patients with PVTT

Table I Summary of Outcomes Following Partial Hepatectomy for HCC-Associated PVTT

\begin{tabular}{|l|l|l|l|l|l|}
\hline Reference & Year & PVTT Classification & Number of Patients & \multicolumn{2}{|c|}{ Outcome } \\
\hline Shi $^{15}$ & 2011 & Type I & 144 & Three-year OS: & $26.70 \%$ \\
& & Type II & $189.90 \%$ \\
& & Type III & 86 & $3.70 \%$ \\
$0 \%$
\end{tabular}


whereupon partial hepatectomy with en bloc PVTT resection would lead to poor outcome measures (five-year overall survival $16-37 \%$ ). The median overall survival was 17.0 vs 7.9 months with a score $\leq 3$ and $>3$, respectively $(p<0.001)$. It remains unclear if this scoring system developed in a cohort of majority HBV related HCC translates to non-HBV etiologies of chronic liver disease.

\section{Portal Venous Tumor Thrombus Beyond the Extent of Hepatic Parenchymal Resection}

For patients presenting with HCC related PVTT with LCSGJ Vp3-4 or Cheng's classification Groups 2-4 (PVTT involving the first-order branches or more proximal or contralateral portal vein), partial hepatectomy with en bloc vascular resection with reconstruction or tumor thrombectomy is surgically feasible, although outcomes and perioperative complications are less favorable than seen with PVTT resected en bloc during partial hepatectomy.

Yamamoto reported five-year disease-free survival and overall survival of Vp3-4 patients of $20.0 \%$ and $30.0 \%$, respectively following partial hepatectomy. ${ }^{23}$ In a retrospective study by Hatano et al, 400 patients with either Vp3 and Vp4 PVTT underwent liver resection with PVTT resection (either with concomitant hepatectomy or tumor thrombectomy). ${ }^{24}$ There was no significant difference in median overall survival (Vp3 24.7 vs Vp4 18.1 months, $\mathrm{p}=0.15$ ) or median disease-free survival (Vp3 6.9 vs Vp4 6.9 months, $\mathrm{p}=0.84$ ). Furthermore, Ban et al detailed HCC patients with Vp3-4 PVTT who underwent liver resection and tumor thrombectomy and found similar survival and recurrence-free survival rates between the two cohorts. ${ }^{25}$ The 1-, 3-, and 5-year survival rates in Vp3 versus Vp4 were 72.0 versus $65.8 \%, 35.3$ versus $41.8 \%$, and 21.2 versus $20.9 \%$, respectively ( $\mathrm{p}=0.821$ ), while the 1-, 3-, and 5-year recurrence-free survival rates in $\mathrm{Vp} 3$ versus $\mathrm{Vp} 4$ were 27.3 versus $35.0 \%, 16.4$ versus $28.0 \%$, and 0 versus $0 \%$, respectively $(\mathrm{p}=0.71)$. Similarly, Ikai et al found no significant survival differences between HCC patients with Vp3 and Vp4 tumor thrombus (fiveyear overall survival, $12 \%$; vs $7 \% ; p=0.438) .{ }^{26,27}$ In all the aforementioned studies the perioperative complication rates remain high with 90-day mortality rates approaching $10 \% .^{23-27}$

Surgical options in $\mathrm{Vp} 3$ and $\mathrm{Vp} 4$ associated $\mathrm{HCC}$ include both partial hepatectomy with tumor thrombectomy and partial hepatectomy with en bloc vascular resection and reconstruction. Several studies have compared the two approaches with regard to survival measures, rates of recurrence, and perioperative morbidity/mortality. Chok et al described the experience at the University of Hong Kong comparing the two surgical approaches. ${ }^{28}$ Although there was no statistically significant difference between the two approaches in either overall survival or disease-free recurrence, the complication rate was much higher (though not statistically significant) in patients undergoing tumor thrombectomy compared to en bloc vascular resection and reconstruction ( $71.4 \%$ vs $50 \%$, respectively). Pleural effusion and severe ascites requiring paracentesis were the most common complications after partial hepatectomy with tumor thrombectomy, and pleural effusion was the most common complication after partial hepatectomy with en bloc vascular resection and reconstruction.

Zhang et al also compared partial hepatectomy with tumor thrombectomy and partial hepatectomy with en bloc vascular resection and reconstruction. ${ }^{29}$ Propensity-score matching was entailed to evaluate 252 patients with type I/II PVTT who underwent liver resection and either en bloc resection of the portal vein for tumor thrombus or a "peeling off" method of thrombectomy. The overall survival and disease-free survival rates were significantly increased in the en bloc group compared with the tumor thrombectomy group ( $\mathrm{p}=0.011$ and $\mathrm{p}=0.015)$ while the tumor thrombectomy cohort had a significantly increased rate of recurrent vascular invasion $(23.9 \%$ vs $9.7 \%$, $\mathrm{p}=0.005)$. There was no significant difference in complications between the two groups. ${ }^{29}$ In contrast to the findings of Zhang et al, the study by Inoue et al found that there was no significant difference between both the 5-year overall and the recurrencefree survival rates in the tumor thrombectomy and the en bloc cohorts $(39 \%$ versus $41 \%$; $p=0.90$ and $23 \%$ versus $18 \% ; \mathrm{p}=0.89)$ though this study included patients with more extensive PVTT ranging from thirdorder branches to the main portal vein. ${ }^{30}$

Obviously, due to the careful selection of patients who would benefit from either of the two approaches, a randomized controlled trial comparing them would not be feasible. As such, the decision of the approach in patients with HCC and extension of PVTT beyond the extent of hepatic parenchymal resection line (LCSG Vp3/Vp4 or Cheng Type II-IV) is largely based on institutional experience. 


\section{Role of Liver Transplantation in Hepatocellular Carcinoma Complicated by Portal Venous Tumor Thrombus}

Liver transplantation (LT) is contraindicated in patients with HCC with PVTT due to the high risk of recurrence and ultimately poor prognosis. ${ }^{31,32}$ Due to the favorable outcomes seen in LT in HCC within the Milan criteria, ${ }^{33}$ researchers recently have attempted to expand criteria for $\mathrm{LT}$ in hopes of achieving improved outcomes in more advanced HCC patients especially those complicated by PVTT.

One of the earliest studies suggesting a survival benefit in HCC with PVTT was by Xu and colleagues. ${ }^{34}$ This group compared HCC patients with PVTT who underwent orthotopic LT, surgical resection, and non-surgical treatment. The transplant group had significantly improved 6-month, 1-year, and 2-year OS rates of $66.7 \%, 29.5 \%$ and $23.6 \%$ compared to $33.3 \%, 22.2 \%$, and $14.8 \%$ for the surgical resection group $(\mathrm{p}=0.0335)$ and $42.1 \%, 24.4 \%$ and $4.1 \%$ for the non-surgical group, respectively $(\mathrm{p}=0.0316)$.

Since the study by $\mathrm{Xu}$ in 2004, there have been a few studies attempting to elucidate the appropriate patients to expand the criteria for LT. One such study is by Lee et al who retrospectively analyzed 282 patients that underwent living donor LT for HCC that was complicated by major PVTT (Vp2, Vp3, or Vp4). ${ }^{35}$ The 1-, 3-, and 5-year RFS rates in the entire cohort were $63.6 \%, 45.5 \%$, and $45.5 \%$ with 1 -, 3-, and 5-year OS rates of $72.7 \%, 63.6 \%$, and $63.6 \%$, respectively. Among other risk factors, this group found that PVTT extending to the main portal vein was a significant risk factor for recurrence and worse OS (both $\mathrm{p}<0.01$ ).

Furthermore, Choi et al attempted to identify the degree of vascular invasion that benefitted from living donor LT. ${ }^{36}$ This group retrospectively studied 242 patients: 184 in the control group, 24 in the microvascular invasion (MVI; Vp1 or Vp2) group, and 34 in the PVTT (Vp3) group after undergoing living donor LT. The DFS and OS were significantly worse in those patients with PVTT. The 1-, 3-, and 5 -year DFS rates were $59.5 \%, 52.9 \%$, and $52.9 \%$, respectively, in the PVTT group whereas the rates were $78.2 \%$, $72.6 \%$, and $64.5 \%$ respectively, in the MVI group $(\mathrm{p}<$ 0.001 ). Similarly, the 1-, 3-, and 5-year OS rates were also significantly worse in the PVTT group than in the MVI group at $78.9 \%, 69.7 \%$, and $63.3 \%$, respectively, compared to $82.2 \%, 50.2 \%$, and $42.5 \%$, respectively ( $p<0.001)$. The recurrence rate in the two-year follow-up period was significantly higher in the PVTT group (44.1\%) compared to the MVI group $(33.3 \%, \mathrm{p}<0.001)$. Choi et al concluded that LT may benefit HCC patients with PVTT; however they proposed even stricter recommendations than Lee et al by only considering those patients with PVTT that has not extended past second-order branches.

While LT has become increasingly utilized for more advanced HCC outside of the Milan criteria including those patients with PVTT, more data is needed regarding long-term outcomes and recurrence rates. An added layer of complexity with the use of LT for advanced HCC is the ethical concerns. Transplant teams must weigh the risks of allocating a limited number of orthotopic grafts or the risks of a major operation to living donors against the benefit of a marginally prolonged survival in a patient with HCC complicated by PVTT.

\section{Conclusions}

Partial hepatectomy with en bloc resection of PVTT in second-order branches and distal (LCSG Vp1/Vp2 or Cheng Type 1) can offer significant benefits in terms of outcome measures and sequelae of portal hypertension in carefully selected patients by experienced centers. Given the relatively poor overall survival and high rates of recurrence combined with the prohibitive perioperative mortality rates, likely secondary to the technical surgical complexity, it remains doubtful whether surgical resection in patients with HCC-associated PVTT extending into first-order portal venous branches or more proximal into the superior mesenteric vein (LCSG Vp3/Vp4 or Cheng Type II-IV) should be offered. Given the development of efficacious systemic and locoregional therapy options surgical resection in patients with HCC and concomitant advanced PVTT should be rarely undertaken. Drawing conclusions from the aforementioned studies is exceedingly difficult due to heterogeneity of both the patient populations and their corresponding clinical outcomes. Though a randomized controlled study is not feasible, HCC patients with PVTT should be included in future comparative studies of neoadjuvant or adjuvant therapy followed by surgical resection to continue assess whether these therapeutic strategies are beneficial.

\section{Funding}

Supported with funding by: 1R01MD012565-01 (AY and $\mathrm{NN})$.

\section{Disclosure}

The authors report no conflicts of interest in this work. 


\section{References}

1. Sung H, Ferlay J, Siegel RL, et al. Global cancer statistics 2020: GLOBOCAN estimates of incidence and mortality worldwide for 36 cancers in 185 countries. CA Cancer J Clin. 2021;71:209-249.

2. Siegel RL, Miller KD, Fuchs HE, Jemal A. Cancer statistics, 2021. CA Cancer J Clin. 2021;71:7-33.

3. Pang TCY, Lam VWT. Surgical management of hepatocellular carcinoma. World J Hepatol. 2015;7:245-252. doi:10.4254/wjh.v7. i 2.245

4. Fan ST, Lo CM, Liu CL, et al. Hepatectomy for hepatocellular carcinoma: toward zero hospital deaths. Ann Surg. 1999;229:322-330. doi:10.1097/00000658-199903000-00004

5. Collela G, Bottelli R, De Carlis L, et al. Hepatocellular carcinoma: comparison between liver transplantation, resective surgery, ethanol injection, and chemoembolization. Transplant Int. 1998;11:S193196. doi:10.1111/j.1432-2277.1998.tb01113.x

6. Fong Y, Sun RL, Jarnagin WR, Blumgart LH. An analysis of 412 cases of hepatocellular carcinoma at a Western center. Ann Surg. 1999;229:790-800. doi:10.1097/00000658-199906000-00005

7. Cerrito L, Annicchiarico BE, Iezzi R, Gasbarrini A, Pimpili M, Ponziani FR. Treatment of hepatocellular carcinoma in patients with portal vein tumor thrombosis: beyond the known frontiers. World J Gastroenterol. 2019;25:4360-4382. doi:10.3748/wjg.v25. i31.4360

8. Finn RS, Qin S, Ikeda M, et al. Atezolizumab plus bevacizumab in unresectable hepatocellular carcinoma. $N$ Engl $J$ Med. 2020;382:1894-1905. doi:10.1056/NEJMoa1915745

9. Llovet JM, Ricci S, Mazzaferro V, et al. Sorafenib in advanced hepatocellular carcinoma. $N$ Engl J Med. 2008;359:378-390. doi:10.1056/NEJMoa0708857

10. European Association for the Study of the Liver. EASL clinical practice guidelines: management of hepatocellular carcinoma. J Hepatol. 2018;69:182-236.

11. Heimbach JK, Kulik LM, Finn RS, et al. AASLD guidelines for the treatment of hepatocellular carcinoma. Hepatology. 2018;67:358-380. doi:10.1002/hep.29086

12. Mokdad AA, Singal AG, Marrero JA, Zhu H, Yopp AC. Vascular invasion and metastasis is predictive of outcome in Barcelona clinic liver cancer stage C hepatocellular carcinoma. J Natl Compr Canc Netw. 2017;15:197-204. doi:10.6004/jnccn.2017.0020

13. Torzilli G, Belghiti J, Kokudo N, et al. A snapshot of the effective indications and results of surgery for hepatocellular carcinoma in tertiary referral centers: is it adherent to the EASL/AASLD recommendations? An observational study of the HCC East-West Study Group. Ann Surg. 2013;257:929-937. doi:10.1097/ SLA.0b013e31828329b8

14. Liver Cancer Study Group of Japan. The general rules for the clinical and pathological study of primary liver cancer. Jpn J Surg. 1989;19:98-129. doi:10.1007/BF02471576

15. Shi J, Lai ECH, Li N, et al. A new classification for hepatocellular carcinoma with portal vein tumor thrombus. $J$ Hepatobiliary Pancreat Sci. 2011;18:74-80. doi:10.1007/s00534-010-0314-0

16. Xu JF, Liu XY, Wang S, Wen HX. Surgical treatment for hepatocellular carcinoma with portal vein tumor thrombosis: a novel classification. World J Surg Oncol. 2015;13:1-5. doi:10.1186/ s12957-015-0493-x

17. Kumada K, Ozawa K, Okamoto R, et al. Hepatic resection for advanced hepatoceullar carcinoma with removal of portal vein tumor thrombus. Surgery. 1990;108:821-827.
18. House MG, Ito $\mathrm{H}$, Gonen $\mathrm{M}$, et al. Survival after hepatic resection for metastatic colorectal cancer: trends in outcomes for 1,600 patients during two decades at a single institution. $J$ Am Coll Surg. 2010;210:744-752. doi:10.1016/j.jamcollsurg.2009.12.040

19. Kudo M, Izumi N, Kubo S, et al. Report of the 20th Nationwide follow-up survey of primary liver cancer in Japan. Hepatol Res. 2020;50:15-46. doi:10.1111/hepr.13438

20. Kokudo T, Hasegawa K, Matsuyama Y, et al. Survival benefit of liver resection for hepatocellular carcinoma associated with portal vein invasion. $J$ Hepatol. 2016;65:938-943. doi:10.1016/j. jhep.2016.05.044

21. Shi J, Lai ECH, Li N, et al. Surgical treatment of hepatocellular carcinoma with portal vein tumor thrombus. Ann Surg Oncol. 2010;17:2073-2080. doi:10.1245/s10434-010-0940-4

22. Zhang XP, Gao YZ, Chen ZH, et al. An Eastern hepatobiliary surgery hospital/portal vein tumor thrombus scoring system as an aid to decision making on hepatectomy for hepatocellular carcinoma patients with portal vein tumor thrombus: a multicenter study. Hepatology. 2019;69:2076-2090. doi:10.1002/hep.30490

23. Yamamoto Y, Ikoma H, Morimura R, et al. Post-hepatectomy survival in advanced hepatocellular carcinoma with portal vein tumor thrombosis. World J Gastroenterol. 2015;21:246-253. doi:10.3748/ wjg.v21.i1.246

24. Hatano E, Uemoto S, Yamaue H, et al. Significance of hepatic resection and adjuvant hepatic arterial infusion chemotherapy for hepatocellular carcinoma with portal vein tumor thrombus in the first branch of portal vein and the main portal trunk: a project study for hepatic surgery of the Japanese Society of Hepato-BiliaryPancreatic Surgery. $J$ Hepatobiliary Pancreat Sci. 2018;25:395-402.

25. Ban D, Shimada K, Yamamoto Y, et al. Efficacy of a hepatectomy and a tumor thrombectomy for hepatocellular carcinoma with tumor thrombus extending to the main portal vein. $J$ Gastrointest Surg. 2009;13:1921-1928. doi:10.1007/s11605-009-0998-0

26. Ikai I, Yamamoto Y, Yamamoto N, et al. Results of hepatic resection for hepatocellular carcinoma invading major portal and/or hepatic veins. Surg Oncol Clin N Am. 2003;12:65-75. doi:10.1016/S10553207(02)00082-0

27. Ikai I, Yamaoka Y, Yamamoto Y, et al. Surgical intervention for patients with stage IV-A hepatocellular carcinoma without lymph node metastasis. Ann Surg. 1998;227:433-439. doi:10.1097/ 00000658-199803000-00016

28. Chok KSH, Cheung TT, Chan SC, Poon RT, Fan ST, Lo CM. Surgical outcomes in hepatocellular carcinoma patients with portal vein tumor thrombosis. World J Surg. 2014;38:490-496. doi:10.1007/ s00268-013-2290-4

29. Zhang YF, Le Y, Wei W, et al. Optimal surgical strategy for hepatocellular carcinoma with portal vein tumor thrombus: a propensity score analysis. Oncotarget. 2016;7:38845-38856. doi:10.18632/ oncotarget.8642

30. Inoue $\mathrm{Y}$, Hasegawa $\mathrm{K}$, Ishizawa $\mathrm{T}$, et al. Is there any difference in survival according to the portal tumor thrombectomy method in patients with hepatocellular carcinoma? Surgery. 2009;145:9-19. doi:10.1016/j.surg.2008.09.005

31. Sapisochin G, Goldaracena N, Laurence JM, et al. The extended toronto criteria for liver transplantation in patients with hepatocellular carcinoma: a prospective validation study. Hepatology. 2016;64:2077-2088. doi:10.1002/hep.28643

32. Freeman RB. Transplantation for hepatocellular carcinoma: the Milan criteria and beyond. Liver Transplant. 2006;12:S8-S13. doi:10.1002/ 1t.20936 
33. Mazzaferro V, Regalia E, Doci R, et al. Liver transplantation for the treatment of small hepatocellular carcinomas in patients with cirrhosis. $N$ Engl J Med. 1996;334(11):693-699. doi:10.1056/ NEJM199603143341104

34. Xu X, Zheng SS, Liang TB, et al. Orthotopic liver transplantation for patients with hepatocellular carcinoma complicated by portal vein tumor thrombi. Hepatobiliary Pancreat Dis Int. 2004;3:341-344.
35. Lee KW, Suh SW, Choi Y, et al. Macrovascular invasion is not an absolute contraindication for living donor transplantation. Liver Transplant. 2017;23:19-27. doi:10.1002/1t.24610

36. Choi HJ, Kim DG, Na GH, et al. The clinical outcomes of patients with portal vein tumor thrombi after living donor liver transplantation. Liver Transplant. 2017;23:1023-1031. doi:10.1002/ 1t. 24782

\section{Publish your work in this journal}

The Journal of Hepatocellular Carcinoma is an international, peerreviewed, open access journal that offers a platform for the dissemination and study of clinical, translational and basic research findings in this rapidly developing field. Development in areas including, but not limited to, epidemiology, vaccination, hepatitis therapy, pathology and molecular tumor classification and prognostication are al considered for publication. The manuscript management system is completely online and includes a very quick and fair peer-review system, which is all easy to use. Visit http://www.dovepress.com/ testimonials.php to read real quotes from published authors. 